\title{
The Role of Cerium Sites in the Scintillation Mechanism of LSO
}

\author{
J. D. Naud and T. A. Tombrello \\ Division of Physics, Mathematics, and Astronomy, \\ California Institute of Technology, Pasadena, CA 91125 \\ C. L. Melcher and J. S. Schweitzer \\ Schlumberger-Doll Research, Old Quarry Road, Ridgefield, CT 06877-4108
}

\begin{abstract}
The crystal structure of $\mathrm{Lu}_{2}\left(\mathrm{SiO}_{4}\right) \mathrm{O}: \mathrm{Ce}$ ("LSO") has two trivalent cation sites which may be occupied by $\mathrm{Ce}^{3+}$ to form luminescence centers. Previous investigations revealed the existence of two distinct sets of $\mathrm{Ce}^{3+}$ excitation and emission spectra and suggested that the differences in the spectra are due to differences in the crystal fields at the two lattice sites that shift the $5 \mathrm{~d}$ levels of $\mathrm{Ce}^{3+}$. In the present report, we reexamine this issue and present new evidence which suggests a different interpretation. In particular, spectra measured at $13 \mathrm{~K}$ suggest that both lattice sites may give rise to indistinguishable excitation and emission spectra while the second set of observed spectra may arise from $\mathrm{Ce}^{3+}$ located in interstitial sites. The evidence for the interstitial sites is a disappearance of the doublet structure in the emission spectra and a large variation in the population of the sites as a function of total cerium concentration. In addition, the results indicate that the scintillation properties of LSO are influenced by the relative population of $\mathrm{Ce}$ in interstitial sites compared to lattice sites as well as by differences in the transfer efficiencies to different sites.
\end{abstract}

\section{INTRODUCTION}

The host material of LSO is $\mathrm{Lu}_{2}\left(\mathrm{SiO}_{4}\right) \mathrm{O}$, which has a monoclinic structure of space group $\mathrm{C} 2 / \mathrm{c}$, and is a good insulator, with a band gap energy of approximately $6 \mathrm{eV}$. In this lattice, the Lu atoms occupy two equally-populated, crystallographically independent sites, which have oxygen coordination numbers of 6 and 7 , and average nearest-neighbor distances of $2.22 \AA$ and $2.32 \AA$, respectively [1]. When doped with cerium, the $\mathrm{Ce}$ is assumed to substitute for the $\mathrm{Lu}$. Ce $\mathrm{Ce}^{3+}$ possesses a single valence electron in the $4 f$ level, which can be excited into the $5 \mathrm{~d}$ level. The $5 \mathrm{~d}$ level is split by the crystal field of the host lattice into 3 sublevels, and the $4 f$ ground state is split by the spin-orbit interaction into two levels, with an energy separation of $2253 \mathrm{~cm}^{-1}[2]$. The luminescence of LSO is due to parity-allowed electric dipole transitions from the lowest $5 \mathrm{~d}$ sublevel to the split $4 \mathrm{f}$ ground state.

Under UV-excitation LSO exhibits two distinct types of excitation and emission spectra. Since there are two crystallographically independent lutetium sites in the host lattice into which the $\mathrm{Ce}$ can substitute, a two-activationcenter model has been proposed in which the two sets of excitation and emission spectra are identified with $\mathrm{Ce}^{3+}$ substituted at the two different Lu sites, designated $\mathrm{Cel}$ and
$\mathrm{Ce} 2$ [3].

The motivation for the present investigation is the variation observed in the scintillation and luminescent properties of LSO crystals produced under a variety of growth conditions and with different cerium concentrations. Although LSO crystals grown under optimal conditions and with optimal cerium concentrations have high light output $(>20,000 \mathrm{ph} / \mathrm{MeV})$, the light output of other LSO crystals may be as much as an order of magnitude less. In addition to the total room temperature light output, the temperature dependence of the gamma-ray-excited light output is observed to vary from crystal to crystal.

\section{EXPERIMENT}

All the samples used in this study were grown by the Czochralski technique which is described elsewhere [4]. The concentration of $\mathrm{Ce}$ in the melts from which the crystals were grown varied from boule to boule. The distribution coefficient of Ce in LSO is relatively low, approximately 0.22 , due to the large difference in ionic radii between the host $\mathrm{Lu}^{3+}(0.848 \AA)$ and the dopant $\mathrm{Ce}^{3+}(1.034 \AA)$ [4]. Since the experimentally determined distribution coefficient is relatively uniform over a wide range of $\mathrm{Ce}$ melt concentrations, we assume that for all LSO samples the concentration of $\mathrm{Ce}$ in a given crystal is proportional to the concentration of $\mathrm{Ce}$ in the corresponding melt.

The set of samples chosen for this study is given in Table 1. The light output is from gamma-ray-excitation at room temperature. The seven LSO samples were chosen to represent the extremes in $\mathrm{Ce}$ concentrations and room temperature light outputs observed among all the crystals grown to date.

As noted above, LSO exhibits two distinct types of luminescent spectra, $\mathrm{Ce} 1$ and $\mathrm{Ce} 2$. At temperatures above $\sim 80$ $\mathrm{K}$, thermal broadening causes a significant overlap of the $\mathrm{Ce} 1$ and $\mathrm{Ce} 2$ excitation bands; it becomes difficult to selectively

Table 1. The set of LSO crystals studied.

\begin{tabular}{lll}
\hline Sample & $\begin{array}{l}\text { Ce conc. } \\
\text { (mol \% in melt) }\end{array}$ & Light Output \\
\hline LSO 73 & 0.10 & 2.19 \\
LSO 57 & 0.54 & 1.80 \\
LSO 84 & 0.05 & 1.60 \\
LSO 44 & 0.55 & 1.09 \\
LSO 27 & 0.25 & 0.85 \\
LSO 34 & 2.12 & 0.58 \\
LSO 25 & 0.50 & 0.28
\end{tabular}




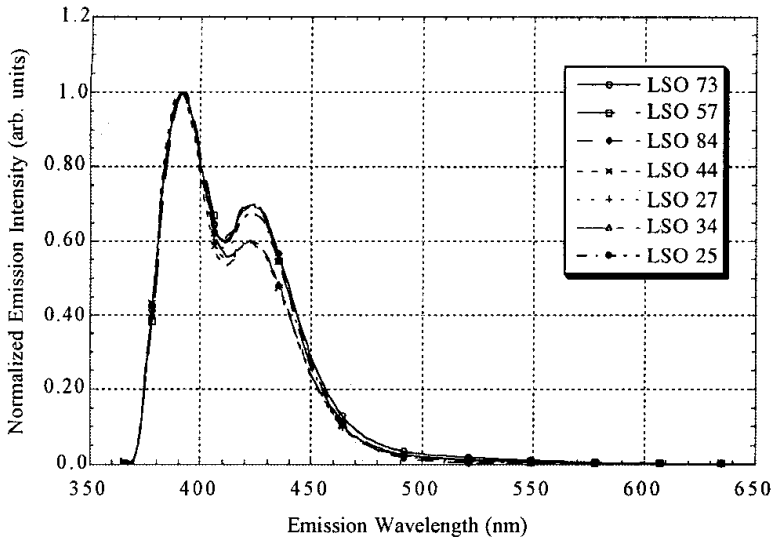

Figure 1. Normalized Cel emission spectra (excited at $356 \mathrm{~nm}$ ) at $13 \mathrm{~K}$. Each spectrum is normalized to its peak intensity.

excite either center independently [3]. Hence, to resolve the two types of luminescence, we performed measurements at low temperature. The crystals $\left(1 \times 1 \times 1 \mathrm{~cm}^{3}\right)$ were mounted on the cold station of a CTI-Cryogenics refrigeration system (Model 21). The temperature was controlled by a nichrome heater wire (NC-32, Lakeshore Electronics) and monitored by a silicon diode sensor (CY7-CU4, OMEGA Electronics) mounted on the copper sample holder. The samples were maintained at a temperature of $13 \pm 1 \mathrm{~K}$ during the measurements. The refrigeration system was incorporated in a SPEX Fluorolog-2 spectrofluorometer. The luminescence was detected with a photomultiplier tube (Hamamatsu R955 with multi-alkali photocathode) used in pulse counting mode. For the UV-excited measurements, the light source was an ozonefree $450 \mathrm{~W}$ high pressure xenon lamp. Double monochrometers were used in both the excitation and emission paths. For the UV-excited measurements, the excitation beam was normally incident on one face of the crystal, and the luminescent emission was measured at an angle of $22.5^{\circ}$ from the normal. For the gamma-ray-excited measurements, the configuration was the same except that an uncollimated $100 \mathrm{mCi}{ }^{241} \mathrm{Am}$ source was placed $\sim 2 \mathrm{~cm}$ from the sample. The band pass was $0.9 \mathrm{~nm}$ for the UV-excited measurements and $2.7 \mathrm{~nm}$ for the gamma-ray-excited measurements.

In addition to these low temperature photoluminescence measurements, we investigated the temperature dependence of the gamma-ray-excited light output of the crystals. Each sample was coupled to a photomultiplier tube (EMR 741Y with high temperature bialkali photocathode and sapphire window), and the assembly was placed inside a temperature controlled chamber along with a $40 \mu \mathrm{Ci}{ }^{137} \mathrm{Cs}$ source. Before any data were taken, each crystal was heated to $150{ }^{\circ} \mathrm{C}$ inside the chamber to empty any thermoluminescent traps, so that any thermoluminescent emission intensity would be small compared with the gamma-ray-excited emission intensity. Measurements of the pulse height distribution were taken at $\sim 25^{\circ} \mathrm{C}$ intervals between $-38^{\circ} \mathrm{C}$ and $150{ }^{\circ} \mathrm{C}$. The data were then corrected for the temperature response of the photomultiplier tube in order to provide the temperature variation of the scintillation efficiency of the crystal.

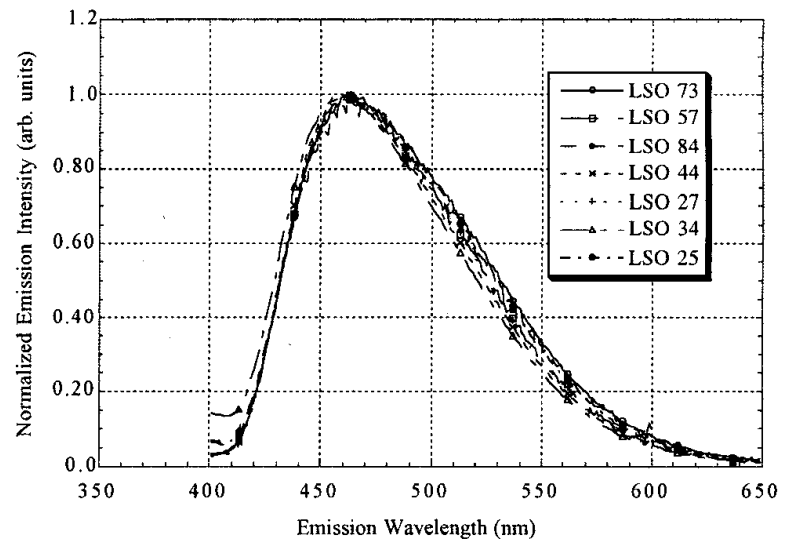

Figure 2. Normalized Ce2 emission spectra (excited at $376 \mathrm{~nm}$ ) at $13 \mathrm{~K}$ Each spectrum is normalized to its peak intensity.

\section{RESULTS}

The Ce1 emission spectrum of each sample was measured at $13 \mathrm{~K}$ using an excitation wavelength of $356 \mathrm{~nm}$, corresponding to the strongest $\mathrm{Cel}$ excitation band [3]. The Cel emission spectra at $13 \mathrm{~K}$, normalized to their peak intensities are given in Figure 1. Emission peaks appear at 393 and $427 \mathrm{~nm}$. The doublet structure of the spectra is due to transitions of the $\mathrm{Ce}^{3+}$ ion from the lowest $5 \mathrm{~d}$ level to the $4 \mathrm{f}$ ground and first excited states [3]. The emission spectra all possess very similar shapes; the only difference between them is the relative height of the lower energy peak.

The $\mathrm{Ce} 2$ emission spectra were measured at $13 \mathrm{~K}$ by exciting the samples at $376 \mathrm{~nm}$, the location of the strongest $\mathrm{Ce} 2$ excitation band; they are shown in Figure 2 normalized to their peak intensities. The $\mathrm{Ce} 2$ spectra are considerably wider than the Cel spectra, having a FWHM of approximately 100 $\mathrm{nm}$ compared to a FWHM of about $60 \mathrm{~nm}$ for Cel. Perhaps more importantly, note that the doublet structure due to the presence of the two $\mathrm{Ce}^{3+}$ 4f states is not observed in these spectra. In addition, there is more variation in the shapes of the normalized $\mathrm{Ce} 2$ spectra than in the shapes of the normalized $\mathrm{Cel}$ spectra.

We assume that the integral of the emission intensity from a given type of $\mathrm{Ce}$ center is directly proportional to the concentration of that type of $\mathrm{Ce}$ center, at least over the range of total cerium concentrations investigated in this study. Therefore, the ratio of the concentrations of the two classes of Ce centers in a sample is proportional to the ratio of the sample's integrated emission intensities from the two types of $\mathrm{Ce}$ centers. For each crystal, we integrated the low temperature, unnormalized $\mathrm{Ce} 1$ and $\mathrm{Ce} 2$ emission spectra and

Table 2. Results for the set of LSO crystals studied.

\begin{tabular}{lllll} 
Sample & $\mathrm{R}_{\mathrm{UV}}$ & $\mathrm{R}_{\mathrm{GR}}$ & $\mathrm{T}_{\mathrm{Q}}(\mathrm{K})$ & $\mathrm{E}_{\mathrm{A}}(\mathrm{eV})$ \\
\hline LSO 73 & 5.67 & 0.487 & 361 & 0.406 \\
LSO 57 & 4.48 & 0.158 & 329 & 0.370 \\
LSO 84 & 24.9 & 0.0975 & 339 & 0.411 \\
LSO 44 & 4.08 & 0.163 & 322 & 0.368 \\
LSO 27 & 4.11 & 0.610 & 318 & 0.365 \\
LSO 34 & 3.18 & 0.147 & 309 & 0.328 \\
LSO 25 & 5.09 & 0.551 & 316 & 0.333 \\
\hline
\end{tabular}




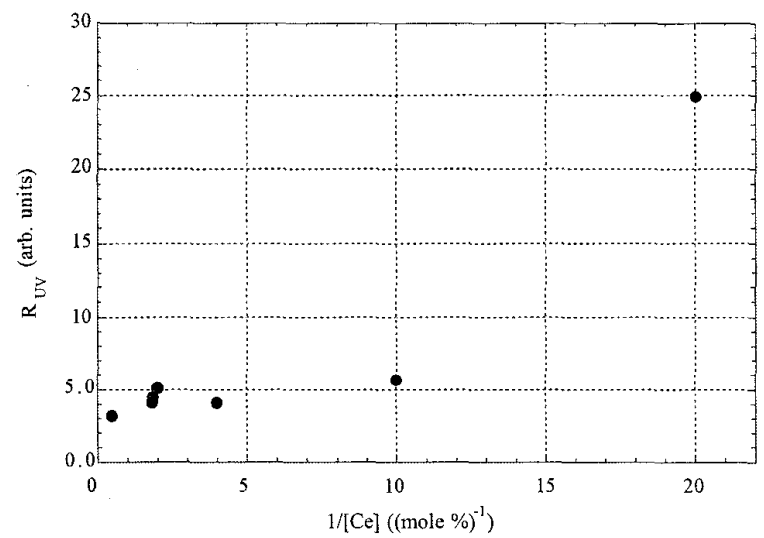

Figure 3. Measure of the ratio of concentrations of $\mathrm{Cel}$ and $\mathrm{Ce} 2$ as a function of the reciprocal of the total $\mathrm{Ce}$ concentration.

took the ratio of the integrals, $\mathrm{R}_{\mathrm{UV}}$, as a measure of $[\mathrm{Ce} 1] /[\mathrm{Ce} 2]$. The results are given in Table 2 . Note that $\mathrm{R}_{\mathrm{UV}}$ varies over a range of almost a factor of eight. In Figure 3 we observe that the ratio of concentrations is correlated with the reciprocal of the total Ce concentration in the crystal.

The gamma-ray-excited emission spectra at $13 \mathrm{~K}$, normalized to their peak intensities, are given in Figure 4 ; two different types of spectral shapes are observed. LSO 34,44 and 57 exhibit considerably more emission above $425 \mathrm{~nm}$ than the other samples in the study. To investigate the cause of this difference, we define $f_{1}(\lambda)$ and $f_{2}(\lambda)$ as the low temperature spectral shape functions for UV-excited $\mathrm{Ce} 1$ and $\mathrm{Ce} 2$ emission, respectively. Based on Figures 1 and 2 we make the approximation that $f_{1}(\lambda)$ and $f_{2}(\lambda)$ are independent of the sample. Given our assumption in the preceding paragraph, the $13 \mathrm{~K} \mathrm{UV}$-excited emission spectrum of $\mathrm{Ce}(\mathrm{i})$ is given by:

$$
\mathrm{UV}_{\mathrm{i}}(\lambda)=\mathrm{k}_{\mathrm{i}}[\mathrm{Ce}(\mathrm{i})] \mathrm{f}_{\mathrm{i}}(\lambda) \quad \mathrm{i}=1,2,
$$

where $\mathrm{k}_{1}$ and $\mathrm{k}_{2}$ are constants, independent of the sample. Similarly, the gamma-ray-excited low temperature spectrum is:

$$
\mathrm{GR}(\lambda)=\mathrm{k}_{3} \eta(\lambda)
$$

where $k_{3}$ is a constant, and $\eta(\lambda)$ is the scintillation efficiency at $13 \mathrm{~K}$. The scintillation efficiency can be written as [5]:

$$
\eta(\lambda)=\beta\left(S_{1} Q_{1}[\operatorname{Ce} 1] f_{1}(\lambda)+S_{2} Q_{2}[\operatorname{Ce} 2] f_{2}(\lambda)\right)
$$

where $\beta$ is the low temperature conversion efficiency, and $S_{1(2)}$ and $Q_{1(2)}$ are the low temperature transfer and luminescent efficiencies for $\mathrm{Ce} 1(2)$, respectively. At $13 \mathrm{~K}$ we assume the quantum efficiency of the luminescent centers to be unity: $Q_{1}=$ $\mathrm{Q}_{2}=1$. Hence, (1), (2) and (3) give:

$$
\mathrm{GR}(\lambda)=\mathrm{C}_{1}\left(\mathrm{R}_{\mathrm{GR}} \mathrm{UV}_{1}(\lambda)+\mathrm{UV}_{2}(\lambda)\right)
$$

where $C_{1}=\left(\beta S_{2} k_{3}\right) / k_{2}$ and

$$
\mathrm{R}_{\mathrm{GR}}=\left(\mathrm{k}_{2} / \mathrm{k}_{1}\right)\left(\mathrm{S}_{1} / \mathrm{S}_{2}\right)
$$

Hence, the quantity $R_{G R}$ is proportional to the ratio of the low

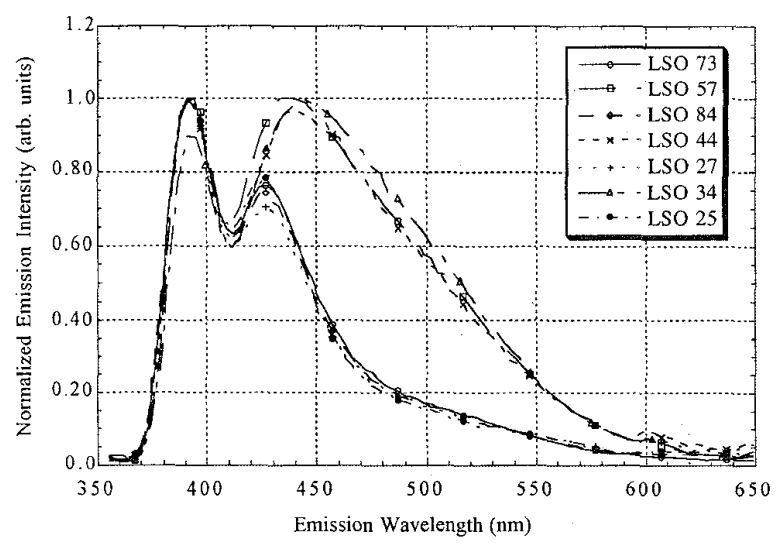

Figure 4. Normalized gamma-ray-excited emission spectra at $13 \mathrm{~K}$. Each spectrum is normalized to its peak intensity.

temperature transfer efficiencies of the two Ce centers. From (4) it is clear that if we fit the gamma-ray-excited emission spectrum of each sample as a weighted sum of the UV-excited $\mathrm{Ce} 1$ and $\mathrm{Ce} 2$ spectra of the sample, the ratio of the best-fit weights will yield $R_{G R}$. An example of the resulting fit, for LSO 44, is given in Figure 5. Note the close agreement between the gamma-ray-excited spectrum and the weighted combination of the UV-excited $\mathrm{Cel}$ and $\mathrm{Ce} 2$ spectra. The agreement for the other samples was at least as good as that demonstrated in Figure 5 . The calculated values of $R_{G R}$ for each sample are given in Table 2 . The results clearly indicate that the low temperature relative transfer efficiency, $S_{1} / S_{2}$, is not uniform among the samples. Based on the values of $\mathrm{R}_{\mathrm{GR}}$ we can divide the LSO samples into three general groups: LSO 84 with $\mathrm{R}_{\mathrm{GR}} \sim 0.1, \mathrm{LSO} 57,44$, and 34 with $\mathrm{R}_{\mathrm{GR}} \sim 0.15$ and LSO 73, 27, and 25 with $\mathrm{R}_{\mathrm{GR}} \sim 0.55$.

We now turn our attention to the temperature dependence of the gamma-ray-excited light output of the samples. The experimental data were fit to a Mott-Gurney equation, which is based on a simple three-level model (ground, excited, and quenching) of the luminescent center [6]:

$$
L(T)=A_{3} /\left(1+A_{1} \exp \left(-A_{2} / T\right)\right),
$$

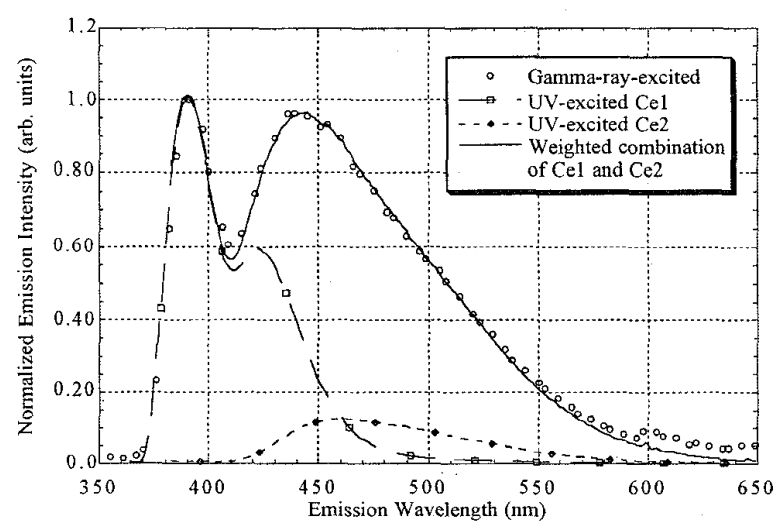

Figure 5. The gamma-ray-excited emission spectrum, the UV-excited $\mathrm{Ce}$ (excited at $356 \mathrm{~nm}$ ) and $\mathrm{Ce} 2$ (excited at $376 \mathrm{~nm}$ ) emission spectrum, and a weighted combination of the $\mathrm{Ce} 1$ and $\mathrm{Ce} 2$ emission spectra for $\mathrm{LSO} 44$ at $13 \mathrm{~K}$. 
where $L$ is the light output, $T$ is the temperature, and $A_{1}, A_{2}$, and $A_{3}$ are parameters of the fit. In this model, $A_{1}$ is a measure of the relative probabilities of nonradiative and radiative transitions from the excited state of the center to the ground state, $A_{2}$ is equal to the energy difference between the excited state and a quenching state (divided by Boltzmann's constant, $\mathrm{k}_{\mathrm{b}}$ ), and $\mathrm{A}_{3}$ is an overall normalization.

The best-fit curves suggest that, below approximately 250 $\mathrm{K}$, the light output becomes essentially independent of temperature. Hence we can use the fit parameters to extrapolate the light output of each crystal to $0 \mathrm{~K}$. From equation (6), the $0 \mathrm{~K}$ light output is $A_{3}$. The extrapolated $0 \mathrm{~K}$ light outputs of the samples obtained in this way are in good agreement with the light outputs at $13 \mathrm{~K}$ obtained by integrating the low temperature gamma-ray-excited emission spectra (shown normalized in Figure 4). This agreement suggests that the fits are reliable, and that the gamma-rayexcited light output is indeed essentially constant below 250 $\mathrm{K}$.

Using the extrapolated $0 \mathrm{~K}$ light outputs, we can normalize the temperature response data for each sample to the $0 \mathrm{~K}$ value and then refit the data with equation (6), this time constraining $A_{3}$ to be 1 . The results are given in Figure 6 . The normalized curves for the LSO samples all possess the same shape; they appear to be just translations of one another. This suggests that the temperature response of each crystal can be approximated by a single parameter. We define this parameter, the quenching temperature, as the temperature at which the crystal's light output is equal to one half its $0 \mathrm{~K}$ value. From equation (6), the quenching temperature, $T_{Q}$, is related to the fit parameters by:

$$
\mathrm{T}_{\mathrm{Q}}=\mathrm{A}_{2} / \log \left(\mathrm{A}_{1}\right)
$$

The calculated quenching temperatures are given in Table 2, along with the activation energies $E_{A}=k_{b} A_{2}$. In Figure 7 we plot the quenching temperature versus $R_{U V}$, the measure of $[\mathrm{Ce} 1] /[\mathrm{Ce} 2]$, for the LSO samples. We observe that, with the exception of LSO 84, $T_{Q}$ appears to be correlated with $R_{U V}$.

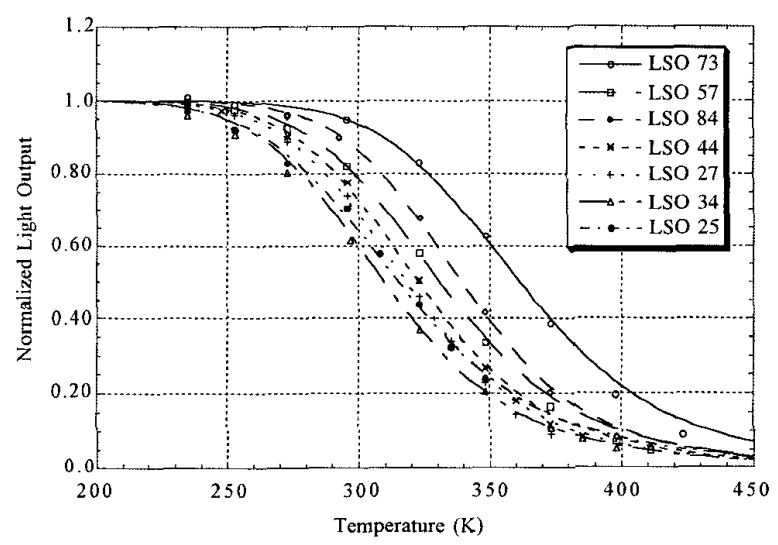

Figure 6. Normalized gamma-ray-excited light output as a function of temperature. The light output for each sample is normalized to 1 at $0 \mathrm{~K}$

\section{DISCUSSION}

The current two-activation-center model of Suzuki et al. of the scintillation mechanism in LSO attributes the two distinct types of UV-excited excitation and emission spectra to $\mathrm{Ce}$ substituted at the two crystallographically independent $\mathrm{Lu}$ sites. We propose an alternate model in which the Cel luminescence is due to Ce substituted at either Lu lattice site, and the $\mathrm{Ce} 2$ luminescence may be due to interstitial Ce centers.

There are several observations in support of this alternate model. First, we clearly observe the spin-orbit splitting of the $\mathrm{Ce}^{3+}$ 4f ground state in the Cel emission spectra, while we see no such splitting in the $\mathrm{Ce} 2$ emission spectra (Figures 1 and 2). If the $\mathrm{Ce} 2$ emission corresponded to $\mathrm{Ce}$ substituted at a definite lattice site, like $\mathrm{Ce} 1$, there is no obvious explanation for the absence of the doublet structure in the low temperature emission spectra. However, if the $\mathrm{Ce} 2$ emission is due to $\mathrm{Ce}$ centers occupying a variety of slightly different interstitial sites, then the variation in the crystal field among these different sites may cause a spread in the energies of the $\mathrm{Ce}^{3+} 5 \mathrm{~d}$ levels. Therefore, the spin-orbit splitting of the ground state in the $\mathrm{Ce} 2$ emission would be "washed-out" because of the range in energy of the lowest $5 \mathrm{~d}$ level. This interpretation is also consistent with the greater width and sample to sample variation seen in the $\mathrm{Ce} 2$ emission spectra as compared with the $\mathrm{Ce} 1$ emission spectra.

A second observation in support of the existence of interstitial Ce centers is the variation seen in $R_{U V}$, which is a measure of $[\mathrm{Ce} 1] /[\mathrm{Ce} 2]$, and its dependence on the total $\mathrm{Ce}$ concentration. Figure 3 indicates that the $\mathrm{Ce}^{3+}$ ions in the melt have a significantly easier time becoming $\mathrm{Ce} 1$ centers than $\mathrm{Ce} 2$ centers, to such a degree that the $\mathrm{Ce} 2$ emission can be essentially eliminated by going to low enough $\mathrm{Ce}$ concentrations, e.g. LSO 84 . This suggests that the difference between the $\mathrm{Ce} 1$ and $\mathrm{Ce} 2$ sites is greater than the difference between the two Lu lattice sites, which differ only slightly in oxygen coordination number ( 6 and 7 ) and by only $\sim 4 \%$ in average nearest-neighbor distance.

Third, recent work-in-progress on the numerical calculation of the energy levels of $\mathrm{Ce}^{3+}$ centers in LSO has found little difference in the $4 \mathrm{f}$ and $5 \mathrm{~d}$ energy levels between the two $\mathrm{Lu}$

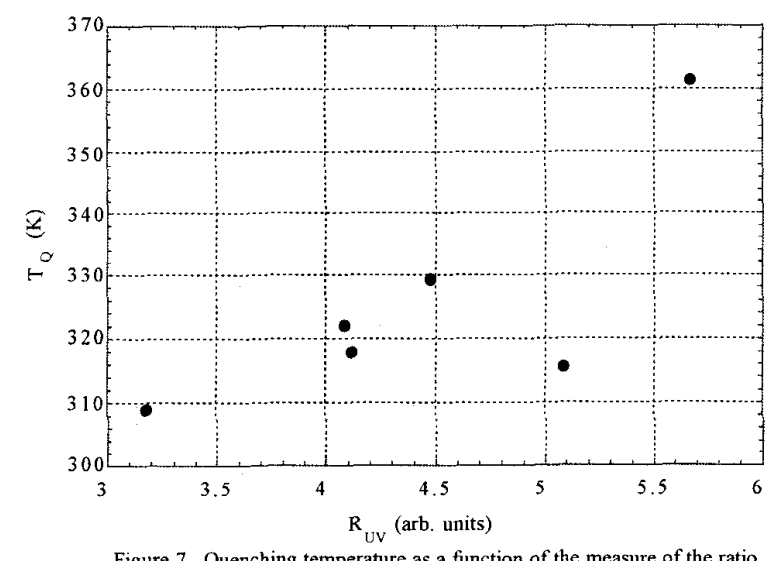
of concentrations of $\mathrm{Ce} 1$ and $\mathrm{Ce} 2$ (LSO 84 not shown). 
lattice sites [1],[7]. This suggests that the luminescent spectra from $\mathrm{Ce}$ at these two types of lattice sites would be very similar, and therefore supports our identification of the Cel spectra as arising from Ce substituted at either Lu lattice site. On the other hand, the spectra in Figure 2 might be consistent with other explanations such as the perturbation of Ce sites by defects such as an oxygen vacancy [8].

Independent of whether the $\mathrm{Ce} 2$ emission is attributed to $\mathrm{Ce}$ at a $\mathrm{Lu}$ lattice site or interstitial $\mathrm{Ce}$, the results of this investigation clearly indicate that both the low temperature ratio of UV-excited integrated emission intensities, $R_{U V}$, and the low temperature ratio of transfer efficiencies, $R_{\mathrm{GR}}$, vary from sample to sample. These variations can be used, to some extent, to explain some of the observed differences in the scintillation properties among the samples. For example, the product $\mathrm{R}_{\mathrm{UV}} \mathrm{R}_{\mathrm{GR}}$, which is proportional to $\left([\mathrm{Ce} 1] \mathrm{S}_{1}\right) /\left([\mathrm{Ce} 2] \mathrm{S}_{2}\right)$ is equal to $\sim 2.4-2.8$ for all the LSO samples except LSO 34 , 44 , and 57 , for which it is $\sim 0.5-0.7$. This result suggests that the difference in shape between the low temperature gammaray-excited spectra of LSO 34,44 , and 57 , and the remaining LSO samples (Figure 4) is due to a combination of the effects of having more $\mathrm{Ce} 2$ relative to $\mathrm{Ce} 1$, and a larger value of $\mathrm{S}_{2}$ relative to $S_{1}$ in these samples, as compared to the other LSO crystals.

The correlation between the quenching temperature of the gamma-ray-excited emission intensity and $\mathrm{R}_{U V}$ (Figure 7) suggests that $\mathrm{Ce} 1$ and $\mathrm{Ce} 2$ centers have different temperature responses, and hence the ratio $[\mathrm{Ce} 1] /[\mathrm{Ce} 2]$ influences the temperature dependence of the overall light output. We note that while LSO 84 is not consistent with the trend visible in Figure 7 , it does possess the highest activation energy of any sample investigated. The reason it does not possess a correspondingly high quenching temperature is that the fit parameter $A_{1}$ is a factor of $\sim 5$ larger for LSO 84 than for any other LSO sample, suggesting that nonradiative processes are more prevalent in LSO 84 than in the other crystals.

\section{ACKNOWLEDGMENTS}

We are grateful to R. A. Manente for growing the crystals used in this study.

\section{REFERENCES}

[1] J. Andriessen, A. Sobolev, A. Kuznetsov, H. Merenga, P. Dorenbos, and C. W. E. van Eijk, "Theoretical investigation of the $4 \mathrm{f}$ and $5 \mathrm{~d}$ levels of cerium in LSO, studied with HF-LCAO and the embedded cluster scattered wave method," to be published.

[2] H. Suzuki, "Scintillation mechanisms of cerium-doped rare earth oxyorthosilicates," California Institute of Technology, Pasadena, CA, Ph.D. dissertation (1994).

[3] H. Suzuki, T. A. Tombrello, C. L. Melcher, and J. S. Schweitzer, "Light emission mechanism of $\mathrm{Lu}_{2}\left(\mathrm{SiO}_{4}\right) \mathrm{O}: \mathrm{Ce}$," IEEE Trans. on Nucl. Science, 40, 380-383 (1993).

[4] C. L. Melcher, R. A. Manente, C. A. Peterson, and J. S. Schweitzer, "Czochralski growth of rare earth oxyorthosilicate single crystals," J. of Crystal Growth, 128, 1001-1005 (1993).

[5] A. Lempicki, A. J. Wojtowicz and E. Berman, "Fundamental limits of scintillator performance," Nucl. Inst. and Meth., A333, 304-311 (1993).

[6] J. B. Birks, The theory and practice of scintillation counting,
New York: Macmillan, 459-460 (1964).

[7] J. Andriessen, P. Dorenbos, C. W. E. van Eijk, "Calculation of energy levels of cerium in inorganic scintillator crystals," Mat. Res. Soc. Symp. Proc., 348, 355 (1994).

[8] C. W. E. van Eijk, personal communication. 